\title{
Generation of Einstein-Podolsky-Rosen-Entangled Radiation through an Atomic Reservoir
}

\author{
Susanne Pielawa, ${ }^{1,2}$ Giovanna Morigi, ${ }^{1}$ David Vitali, ${ }^{3}$ and Luiz Davidovich ${ }^{4}$ \\ ${ }^{1}$ Departament de Fisica, Universitat Autònoma de Barcelona, 08193 Bellaterra, Spain \\ ${ }^{2}$ Institut de Ciències Fotòniques (ICFO), 08860 Castelldefels (Barcelona), Spain \\ ${ }^{3}$ Dipartimento di Fisica, Università di Camerino, 62032 Camerino, Italy \\ ${ }^{4}$ Instituto de Física, Universidade Federal do Rio de Janeiro, 21941-972 Rio de Janeiro, Brazil
}

(Received 25 January 2007; published 12 June 2007)

\begin{abstract}
We propose a scheme for generating two-mode squeezing in high- $Q$ resonators using a beam of atoms with random arrival times, which acts as a reservoir for the field. The scheme is based on four-wave mixing processes leading to emission into two cavity modes, which are resonant with the Rabi sidebands of the atomic dipole transition, driven by a saturating classical field. At steady state the cavity modes are in an Einstein-Podolsky-Rosen state, whose degree of entanglement is controlled by the intensity and the frequency of the transverse field. This scheme is robust against stochastic fluctuations in the atomic beam, does not require atomic detection nor velocity selection, and can be realized by presently available experimental setups with microwave resonators.
\end{abstract}

DOI: $10.1103 /$ PhysRevLett.98.240401

PACS numbers: 03.65.Ud, 03.67.Hk, 42.50.Pq

A single atom is a textbook example of a nonclassical light source. Seminal experiments have shown the sudden nature of spontaneous emission events [1], the quantum properties of the emitted photons [2,3], and entanglement between the atom and its emitted photon [4]. The high degree of control on the dynamics of the interaction between single atoms and the electromagnetic field has allowed access to novel regimes, making single atoms in resonators promising candidates, together with atomic ensembles [5], for implementing interfaces for quantum networks [6]. In optical cavities, for instance, lasing at the single-atom level [7] and controlled single-photon generation [8] have been demonstrated. In the microwave domain remarkable milestones have been achieved thanks to the extremely stable resonators that are available in this frequency domain [9-11]. Some paradigmatic experiments are the preparation and measurement of nonclassical states of the microwave field $[9,10]$, the experimental characterization of loss of coherence of the quantum field [12] and of the transition from quantum to classical dynamics [13], and the quantum nondemolition measurement of the number of photons of the cavity field [14]. In the weak-coupling limit, the atomic beam may act as a reservoir for the cavity mode, leading to thermalization between the beam and the resonator [15]. The opposite regime, with the resonator field saturating the atomic transition, leads, for velocityselected atoms, to sub-Poissonian fields, and, in the weakdissipation limit, to trapping states $[16,17]$. Methods for preparation of an arbitrary single-mode quantum state of the electromagnetic field in a resonator, involving resonant interaction with a well-controlled sequence of atoms, without the need of atomic detection, were proposed in [1820]. Methods requiring atomic-state measurement, on the other hand, rely on high-efficiency detectors, which are lacking in present experiments.
In this Letter, we propose a method for preparing quantum states of the electromagnetic field based on quantum reservoir engineering. This method, so far applied to trapped ions [21], is here implemented in a typical setup of microwave cavity QED as in Fig. 1, where the resonator is pumped by a beam of atoms with random arrival times, and needs neither atomic detection nor detailed control of the sequence of atoms: We show that, by suitably preparing the initial state of the incoming atoms, two-mode squeezing, i.e., Einstein-Podolsky-Rosen (EPR) correlations [22], are established between the cavity modes at steady state.

The underlying mechanism is four-wave mixing, where emission into the cavity modes is enhanced by resonant coupling with the Rabi sidebands induced by a classical field that saturates the atomic transition [23], with the creation of EPR correlations being enforced by the initial

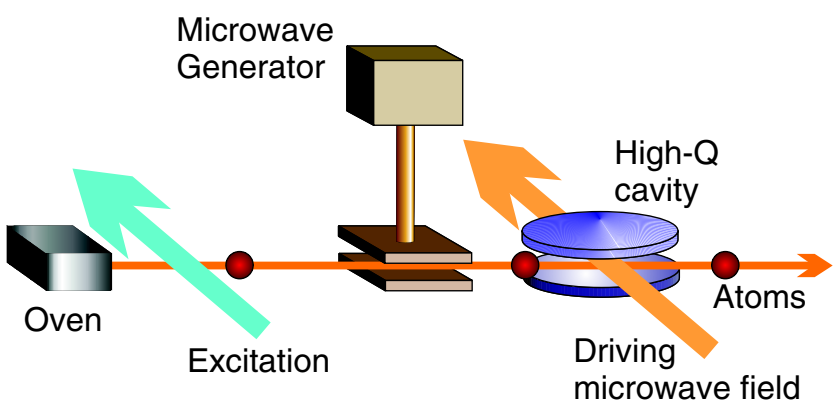

FIG. 1 (color online). Setup of the system. Atoms from a beam are prepared in a coherent superposition of two Rydberg states $|g\rangle$ and $|e\rangle$ by a combination of laser and microwave fields. The atoms have random arrival times, and a low pumping rate warrants that at most one atom is inside the resonator at a time $[9,10]$. While in the cavity, the dipole transition $|g\rangle \rightarrow|e\rangle$ is saturated by a transverse microwave field, thereby pumping on resonance two nondegenerate modes of the resonator, which are led asymptotically to a two-mode squeezed state. 
quantum state of the injected atoms. Contrary to typical setups based on optical parametric amplifiers, the atoms pump the resonator through resonant single-photon processes. Differing from $[19,20]$, the atoms do not need to be initially correlated nor does their number have to be controlled: The atomic beam acts as a reservoir, where the atoms interact with the field one at a time [24], and that pulls the field into the desired state. The degree of entanglement is controlled by the intensity of the laser and its detuning from the atomic transition. Many photons per mode can be achieved in accessible experimental regimes. Control on atomic velocity (interaction time) and atomic detection are not required. The scheme is robust against stochastic fluctuations in the atomic beam. This method may constitute an important step towards the implementation of quantum networking with continuous variables [25] in the microwave regime.

The basic system is sketched in Fig. 1. Prior to the interaction region, the atoms are prepared in a coherent superposition of two Rydberg states $|g\rangle$ and $|e\rangle$ connected by a dipole transition. For an open-cavity geometry [9], an electric potential between the two mirrors removes through Stark shifting the degeneracy of circular Rydberg states. Inside the resonator a classical field saturates the dipole transition, thereby pumping on resonance two nondegenerate modes of the resonator at frequencies $\omega_{1}$ and $\omega_{2}$, as sketched in Fig. 2. The corresponding Hamiltonian is similar to the one describing four-wave mixing as in [23], and has the form

$$
\begin{aligned}
\hat{H}= & \hbar \omega_{0} \hat{\sigma}^{\dagger} \hat{\sigma}+\hbar \Omega\left(e^{-i \omega_{L} t} \hat{\sigma}^{\dagger}+e^{i \omega_{L} t} \hat{\sigma}\right) \\
& +\sum_{\lambda}\left[\hbar \omega_{\lambda} \hat{a}_{\lambda}^{\dagger} \hat{a}_{\lambda}+\hbar g_{\lambda}\left(\hat{a}_{\lambda} \hat{\sigma}^{\dagger}+\hat{a}_{\lambda}^{\dagger} \hat{\sigma}\right)\right]
\end{aligned}
$$

where $\omega_{0}$ is the transition frequency, $\hat{a}_{\lambda}$ and $\hat{a}_{\lambda}^{\dagger}$ are photon annihilation and creation operators for the mode with a)

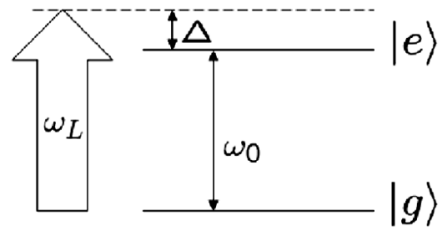

b)

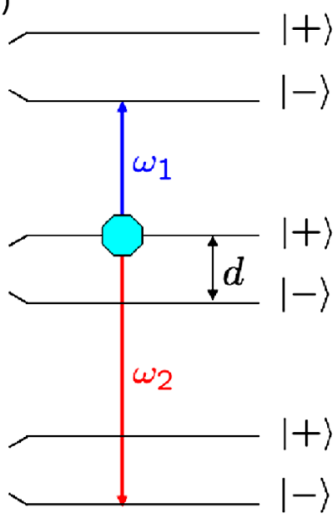

FIG. 2 (color online). (a) Dipole transition $|g\rangle \rightarrow|e\rangle$ at frequency $\omega_{0}$ coupling to the classical field at frequency $\omega_{L}$. (b) Resulting ladder of the semiclassical dressed states $| \pm\rangle$. A transition $|+\rangle \rightarrow|-\rangle$ is accompanied by absorption (emission) of a photon of frequency $\omega_{1}\left(\omega_{2}\right)$, and vice versa; see text. frequency $\omega_{\lambda}(\lambda=1,2), g_{\lambda}$ are the coupling constants between the two-level atom and each cavity mode, and $\hat{\sigma}^{\dagger}=|e\rangle\langle g|, \hat{\sigma}=| g\rangle\langle e|$ are the atomic raising and lowering operators [26]. The time-dependent term describes the coupling, with strength $\Omega$, between the dipole and the external classical field at frequency $\omega_{L}$.

The transformation $\hat{U}=\exp \left[i \omega_{L} t\left(\hat{\sigma}^{\dagger} \hat{\sigma}+\sum_{\lambda} \hat{a}^{\dagger} \hat{a}\right)\right]$ takes the above Hamiltonian into the rotating-frame form

$$
\hat{H}^{\mathrm{RF}}=\hat{\mathcal{H}}_{0}-\sum_{\lambda} \hbar \delta_{\lambda} \hat{a}_{\lambda}^{\dagger} \hat{a}_{\lambda}+\sum_{\lambda} \hbar g_{\lambda}\left(\hat{\sigma}^{\dagger} \hat{a}_{\lambda}+\hat{\sigma} \hat{a}_{\lambda}^{\dagger}\right),
$$

where $\delta_{\lambda}=\omega_{L}-\omega_{\lambda}$, and $\hat{\mathcal{H}}_{0}=-\hbar \Delta \hat{\sigma}^{\dagger} \hat{\sigma}+\hbar \Omega\left(\hat{\sigma}^{\dagger}+\right.$ $\hat{\sigma})$ describes the coupling between dipole and classical field, with detuning $\Delta=\omega_{L}-\omega_{0}$; see Fig. 2(a). We assume the coupling to the driving field to be much stronger than the coupling to the cavity modes, $|\Omega| \gg\left|g_{\lambda}\right|$, and express Hamiltonian (2) in the basis of eigenstates $| \pm\rangle$ of $\hat{\mathcal{H}}_{0}, \quad$ with $\quad \hat{\mathcal{H}}_{0}| \pm\rangle=-\hbar(\Delta \mp d) / 2| \pm\rangle$ and $d=$ $\sqrt{\Delta^{2}+4 \Omega^{2}}$. These are the semiclassical dressed states, with $|+\rangle=\sin \theta|g\rangle+\cos \theta|e\rangle,|-\rangle=\cos \theta|g\rangle-\sin \theta|e\rangle$, and $\tan \theta=2|\Omega| /(d-\Delta)$. The corresponding energy levels are shown in Fig. 2(b). We denote the raising and lowering operators in the new basis by $\hat{\pi}^{+}=|+\rangle\langle-|$and $\hat{\pi}^{-}=|-\rangle+\mid$, with $\hat{\pi}_{z}=|+\rangle\langle+|-|-\rangle\langle-|$, and write $\hat{H}^{\mathrm{RF}}=\hat{H}_{0}+\hat{H}_{\text {int }}$, with $\hat{H}_{0}=\hbar d \hat{\pi}_{z} / 2-\hbar \sum_{\lambda} \delta_{\lambda} \hat{a}_{\lambda}^{\dagger} \hat{a}_{\lambda}$ and

$$
\begin{aligned}
\hat{H}_{\mathrm{int}}= & \sum_{\lambda} \hbar g_{\lambda}\left[\hat{\pi}_{z}\left(\hat{a}_{\lambda}+\hat{a}_{\lambda}^{\dagger}\right) \cos \theta \sin \theta\right. \\
& \left.+\left(\hat{\pi}^{+} \hat{a}_{\lambda}+\hat{a}_{\lambda}^{\dagger} \hat{\pi}^{-}\right) \cos ^{2} \theta-\left(\hat{\pi}^{-} \hat{a}_{\lambda}+\hat{\pi}^{+} \hat{a}_{\lambda}^{\dagger}\right) \sin ^{2} \theta\right] .
\end{aligned}
$$

If $\left|g_{\lambda}\right| \ll d$ we can choose which processes are resonant, and thus relevant for the dynamics, by changing the values of $\delta_{1}, \delta_{2}$, and $d$. One is thus able to generate a diversity of dynamical processes. For instance, generation of cat states of a single-mode resonator, as discussed in Ref. [27], is recovered from $\hat{H}^{\mathrm{RF}}$ by setting $d=-\delta_{1}$ and $\left|\delta_{2}\right| \gg$ $d,\left|\delta_{1}\right|, g_{\lambda}$, hence obtaining $\hat{H}^{\mathrm{RF}} \approx \hbar g_{1} \hat{\pi}_{z}\left(\hat{a}_{1}+\hat{a}_{1}^{\dagger}\right)$. The Hamiltonian of the optical parametric amplifier is obtained from $\hat{H}$ by selecting resonant two-photon processes and off-resonant single-photon transitions. The latter, however, produce ac-Stark shifts which limit the efficiency of such a scheme. Instead, we propose here the generation of robust two-mode squeezed states using resonant single-photon processes.

These single-photon processes are selected by setting $\delta_{1}=d$ and $\delta_{2}=-d$; see Fig. 2(b). If $\left|g_{\lambda}\right| \ll d$ we obtain from Eq. (3) the effective Hamiltonian $\hat{H}^{\mathrm{RF}} \approx \hat{H}_{\text {eff }}$, with

$$
\hat{H}_{\text {eff }}=\hat{H}_{0}+\left[\hbar g\left(\hat{a}_{2}^{\dagger} \cos ^{2} \theta-\hat{a}_{1} \sin ^{2} \theta\right) \hat{\pi}^{-}+\text {H.c. }\right] \text {, }
$$

where $\hat{H}_{0}=\hbar d\left(\hat{\pi}_{z} / 2-\hat{a}_{1}^{\dagger} \hat{a}_{1}+\hat{a}_{2}^{\dagger} \hat{a}_{2}\right)$ and we have assumed $g:=g_{1}=g_{2}$. The processes described by Eq. (4) are indicated by the arrows in Fig. 2(b). Using the twomode squeezing operator $\hat{S}\left(r_{\mu}\right)=\exp \left(r_{\mu}^{*} \hat{a}_{1} \hat{a}_{2}-r_{\mu} \hat{a}_{1}^{\dagger} \hat{a}_{2}^{\dagger}\right)$ 
we bring Hamiltonian (4) to the well-known JaynesCummings form, $\hat{S}^{\dagger}\left(r_{\mu}\right) \hat{H}_{\text {eff }} \hat{S}\left(r_{\mu}\right)=\hat{H}_{0}+\hat{H}_{\text {int }}$, with $\hat{H}_{0}=\hbar d\left(\hat{\pi}_{z} / 2-\hat{b}_{1}^{\dagger} \hat{b}_{1}+\hat{b}_{2}^{\dagger} \hat{b}_{2}\right)$ and

$$
\begin{array}{cc}
\hat{H}_{\mathrm{int}}=-\hbar \Omega_{b}\left(\hat{b}_{1} \hat{\pi}^{-}+\hat{b}_{1}^{\dagger} \hat{\pi}^{+}\right), & \text {if } \Delta>0, \\
\hat{H}_{\mathrm{int}}=\hbar \Omega_{b}\left(\hat{b}_{2}^{\dagger} \hat{\pi}^{-}+\hat{b}_{2} \hat{\pi}^{+}\right), & \text {if } \Delta<0 .
\end{array}
$$

Here, $\Omega_{b}=g \sqrt{(1-\mu) /(1+\mu)}$ with $r_{\mu}=\operatorname{arctanh} \mu$, while the value of $\mu$ is determined by the classical field parameters, $\mu=\tan ^{2} \theta$ if $|\tan \theta|<1$, otherwise $\mu=$ $(\tan \theta)^{-2}$ if $|\tan \theta|>1$. The new bosonic operators $\hat{b}_{1}, \hat{b}_{2}$, are connected to $\hat{a}_{1}$ and $\hat{a}_{2}$ by the two-mode squeezing transformation, $\hat{b}_{j}=\hat{S}^{\dagger}\left(r_{\mu}\right) \hat{a}_{j} \hat{S}\left(r_{\mu}\right)$. The sign of the detuning $\Delta$ determines to which of the transformed modes the two-level transition couples [28]. Let $\left|n_{1}, n_{2}\right\rangle_{a}$ and $\left|n_{1}, n_{2}\right\rangle_{b}$ be the eigenvectors of the number operators $\hat{a}_{j}^{\dagger} \hat{a}_{j}$ and $\hat{b}_{j}^{\dagger} \hat{b}_{j}$, respectively, corresponding to the eigenvalues $n_{j}=$ $0,1,2, \ldots(j=1,2)$. The two bases are related by the transformation $\left|n_{1}, n_{2}\right\rangle_{a}=\hat{S}\left(r_{\mu}\right)\left|n_{1}, n_{2}\right\rangle_{b}$. In particular, the vacuum state in the $b$ basis is a two-mode squeezed state of the two cavity modes, $|0,0\rangle_{b}=\hat{S}^{\dagger}\left(r_{\mu}\right)|0,0,\rangle_{a}$, with degree of squeezing determined by $\mu$ and thus by the ratio $|\Delta / \Omega|$. For $\Delta>0(\Delta<0)$ the state $|+, 0,0\rangle_{b}$ $\left(|-, 0,0\rangle_{b}\right)$ is the ground state of the new Hamiltonian.

We now show how the cavity modes can be prepared in the two-mode squeezed state asymptotically. This is achieved by an effective "dissipation" process in the $b$ basis, implemented in a two-step procedure sketched in Fig. 3(a). Step 1: One sets $\Delta=\Delta_{0}>0$. The atoms enter the cavity in state $|+\rangle$ and undergo the dynamics of Eq. (5), removing in average excitations from mode $b_{1}$. Step 2: Dynamics of Eq. (6) is selected by setting $\Delta=-\Delta_{0}$. The atoms enter in the state $|-\rangle$ and absorb in average excitations from mode $\hat{b}_{2}$.

We assume the weak-coupling regime, where the interaction of a single atom with the cavity is a small perturba- tion [29]. Let $\tau$ be the interaction time, with $\Omega_{b} \tau \ll 1$, and let all atoms be initially prepared in state $|+\rangle$ in step 1 , and in state $|-\rangle$ in step 2 . The differential change on the density matrix $\hat{\rho}_{t}$ of the cavity during each step $j(j=1,2)$ is [15]

$$
\left.\frac{\partial \hat{\rho}_{t}}{\partial t}\right|_{\text {step j }}=-\frac{\gamma}{2}\left(\hat{b}_{j}^{\dagger} \hat{b}_{j} \hat{\rho}_{t}-2 \hat{b}_{j} \hat{\rho}_{t} \hat{b}_{j}^{\dagger}+\hat{\rho}_{t} \hat{b}_{j}^{\dagger} \hat{b}_{j}\right),
$$

where $\gamma=r_{\mathrm{at}} \Omega_{b}^{2} \tau^{2}$ and $r_{\mathrm{at}}$ is the atomic arrival rate. Hence, during step $j$ we have $\left\langle\hat{b}_{j}^{\dagger} \hat{b}_{j}\right\rangle_{t}=\left\langle\hat{b}_{j}^{\dagger} \hat{b}_{j}\right\rangle_{0} \exp (-\gamma t)$, which vanishes at times $t \gg 1 / \gamma$; see Fig. 3(a). In terms of the original field modes, this procedure implies that the atoms pump in phase only the two-mode squeezed state. Asymptotically, the field state approaches

$$
\hat{\rho}_{\infty}=|0,0\rangle_{b}\left\langle 0,0\left|=\hat{S}^{\dagger}\left(r_{\mu}\right)\right| 0,0\right\rangle_{a}\langle 0,0| \hat{S}\left(r_{\mu}\right)
$$

which is a two-mode squeezed state, whose degree of squeezing $r_{\mu}$ is solely determined by the ratio $|\Delta / \Omega|$. This state is reached independently of the initial state of the cavity modes, provided that each step is implemented for a sufficiently long time $T$.

We now analyze the proposal requirements. The twostep procedure needs a change in the transition frequency of the two-level atom, which can be achieved by an external static field. The scheme needs neither atomic detection nor control of the number of atoms nor of the interaction times (atomic velocities). On the other hand, the atom must not decay during the interaction with the cavity modes, and dissipation of the cavity field should be negligible during the experiment. Experiments with microwave resonators $[9,10]$ are characterized by interaction times of the order of tens of microseconds, which warrant negligible spontaneous decay, typically of the order of tens of milliseconds. The time $T$ required for each step to reach $\left\langle\hat{b}_{j}^{\dagger} \hat{b}_{j}\right\rangle_{T} \sim \bar{n}_{\infty}$ depends on the initial value $\left\langle\hat{b}_{j}^{\dagger} \hat{b}_{j}\right\rangle_{0}=: \bar{n}_{0}$ through $T=\gamma^{-1}\left|\log \left(\bar{n}_{\infty} / \bar{n}_{0}\right)\right|$. Figure 3(b) displays the

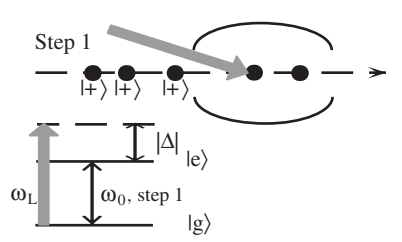

a)

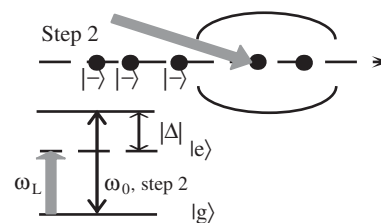

b)

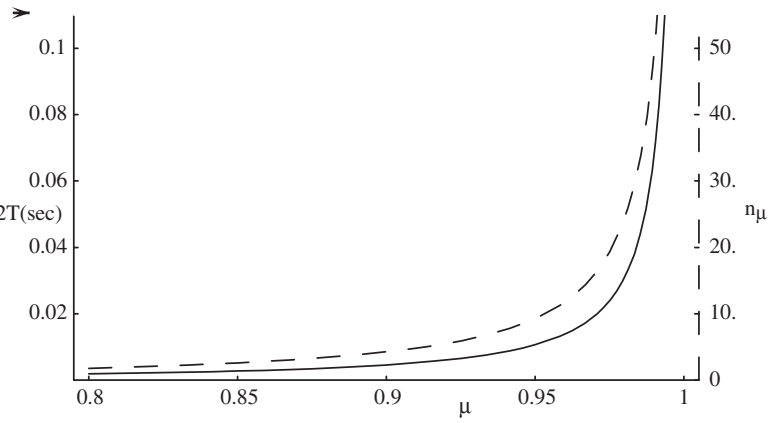

FIG. 3. (a) Schematic representation of the two-step procedure. In the plot, the expectation values of the operators $\hat{b}_{1}^{\dagger} \hat{b}_{1}$ and $\hat{b}_{2}^{\dagger} \hat{b}_{2}$ are shown as a function of time, in units of $\tau_{0}=\gamma^{-1}$, for $\mu=0.97$. After a sufficiently long time $T$ the steady state $|0,0\rangle_{b}$, corresponding to the two-mode squeezed state of the cavity modes, is reached with fidelity $F=0.98$. (b) Average photon number (dashed line) and total experimental time $T$, in seconds, as a function of $\mu$. 
estimated total experimental times and corresponding average number of photons per mode at steady state as a function of $\mu$, where $\bar{n}_{0}=\mu^{2} /\left(1-\mu^{2}\right)$ when the cavity modes are in the vacuum state at $t=0$. For the degree of squeezing $r_{\mu} \approx 2.1(\mu=0.97)$, leading to an average number of 16 photons per mode at steady state, and $\bar{n}_{\infty}=$ 0.01 , corresponding to a fidelity $F \approx 0.98$, then one has $2 T \sim 19 \mathrm{~ms}$ in case of an initially empty cavity $(2 T \sim$ $22 \mathrm{~ms}$ for 0.7 thermal photons). Resonators stable over this time are available in present experiments [11]. Fluctuations in the coupling with the driving field $\delta \Omega$ give rise to an effective linewidth of the dressed states, and are negligible provided that $\delta \Omega T \ll 1$ during $T$. This holds if the coherence time of the driving field is much larger than $T$, which is easily achievable with current microwave sources. Statistical properties of the cavity field can be evaluated by measuring the internal states of the emerging atoms [30]. Its state can also be determined by reconstructing the corresponding Wigner function, by suitably generalizing the schemes proposed in [31].

In conclusion, a highly nonclassical state of radiation can be generated in a cavity as the steady state of the stochastic interaction with a beam of two-level atoms. This final outcome does not depend on the initial state of the field. The atoms constitute a spin reservoir, which plays no detrimental role for quantum coherence, but it assists its formation, leading to an EPR-like state of the electromagnetic field. In this sense, this is an instance of reservoir engineering within the framework of cavity quantum electrodynamics. Because of its robustness, this proposal is an important step towards quantum networking with atomphoton interfaces in the microwave regime.

We acknowledge discussions with M. Brune, K. Eckert, J. Eschner, M. Hennrich, and S. Stenholm, and support by the European commission (SCALA, Contract No. 015714; EMALI, No. MRTN-CT-2006-035369), the Spanish MEC (Consolider Ingenio 2010 "QOIT"; QLIQS, No. FIS200508257-C02-01; Ramon-y-Cajal), the Carl-Duisberg foundation, the Brazilian agencies CNPq, FAPERJ, FUJB, and the Brazilian Millennium Institute for Quantum Information.

[1] W. Nagourney, J. Sandberg, and H. Dehmelt, Phys. Rev. Lett. 56, 2797 (1986); Th. Sauter, W. Neuhauser, R. Blatt, and P.E. Toschek, Phys. Rev. Lett. 57, 1696 (1986).

[2] H. J. Kimble, M. Dagenais, and L. Mandel, Phys. Rev. Lett. 39, 691 (1977); F. Diedrich and H. Walther, Phys. Rev. Lett. 58, 203 (1987).

[3] T. Legero et al., Phys. Rev. Lett. 93, 070503 (2004); J. Beugnon et al., Nature (London) 440, 779 (2006).

[4] B. Blinov, D. L. Moehring, L.-M. Duan, and C. Monroe, Nature (London) 428, 153 (2004); J. Volz et al., Phys. Rev. Lett. 96, 030404 (2006).
[5] See, for instance, J. F. Sherson et al., Nature (London) 443, 557 (2006).

[6] M. D. Lukin, Rev. Mod. Phys. 75, 457 (2003).

[7] K. An, J. J. Childs, R. R. Dasari, and M. S. Feld, Phys. Rev. Lett. 73, 3375 (1994); J. McKeever et al., Nature (London) 425, 268 (2003).

[8] A. Kuhn, M. Hennrich, and G. Rempe, Phys. Rev. Lett. 89, 067901 (2002); J. McKeever et al., Science 303, 1992 (2004); M. Keller et al., Nature (London) 431, 1075 (2004).

[9] J. M. Raimond, M. Brune, and S. Haroche, Rev. Mod. Phys. 73, 565 (2001).

[10] H. Walther, B. T. H. Varcoe, B.-G. Englert, and T. Becker, Rep. Prog. Phys. 69, 1325 (2006).

[11] S. Kuhr et al., arXiv:quant-ph/0612138.

[12] M. Brune et al., Phys. Rev. Lett. 77, 4887 (1996).

[13] A. Auffeves et al., Phys. Rev. Lett. 91, 230405 (2003); T. Meunier et al., Phys. Rev. Lett. 94, 010401 (2005).

[14] S. Gleyzes et al., Nature (London) 446, 297 (2007).

[15] B.-G. Englert and G. Morigi, in Coherent Evolution in Noisy Environments, edited by A. Buchleitner and K. Hornberger (Springer, Berlin, 2002), p. 55.

[16] P. Filipowicz, J. Javanainen, and P. Meystre, Phys. Rev. A 34, 3077 (1986).

[17] M. Weidinger, B.T.H. Varcoe, R. Heerlein, and H. Walther, Phys. Rev. Lett. 82, 3795 (1999).

[18] A. S. Parkins, P. Marte, P. Zoller, and H. J. Kimble, Phys. Rev. Lett. 71, 3095 (1993).

[19] C. K. Law and J.H. Eberly, Phys. Rev. Lett. 76, 1055 (1996).

[20] Th. Wellens, A. Buchleitner, B. Kümmerer, and H. Maassen, Phys. Rev. Lett. 85, 3361 (2000).

[21] J. F. Poyatos, J. I. Cirac, and P. Zoller, Phys. Rev. Lett. 77, 4728 (1996); A. R. R. Carvalho, P. Milman, R. L. de Matos Filho, and L. Davidovich, Phys. Rev. Lett. 86, 4988 (2001); C. J. Myatt et al., Nature (London) 403, 269 (2000).

[22] A. Einstein, B. Podolsky, and N. Rosen, Phys. Rev. 47, 777 (1935); M. D. Reid, Phys. Rev. A 40, 913 (1989).

[23] This process is described in G. S. Agarwal and R. W. Boyd, Phys. Rev. A 38, 4019 (1988).

[24] A similar model has been discussed in V. Scarani et al., Phys. Rev. Lett. 88, 097905 (2002).

[25] S. L. Braunstein and P. van Loock, Rev. Mod. Phys. 77, 513 (2005).

[26] The field's polarizations must be such that they all couple with the dipole transition $|g\rangle \rightarrow|e\rangle$, while coupling to other states is avoided. For Stark-shifted circular Rydberg states, this can be achieved by using circular polarizations for the cavity modes and for the pump field.

[27] E. Solano, G. S. Agarwal, and H. Walther, Phys. Rev. Lett. 90, 027903 (2003).

[28] At $\Delta=0$ no correlations between the cavity modes are established by this interaction.

[29] The same steady state can also be achieved in the strongcoupling regime by randomizing the interaction times.

[30] H. J. Briegel, B.-G. Englert, N. Sterpi, and H. Walther, Phys. Rev. A 49, 2962 (1994).

[31] L. G. Lutterbach and L. Davidovich, Phys. Rev. Lett. 78, 2547 (1997); P. Lougovski et al., Phys. Rev. Lett. 91, 010401 (2003). 